\title{
OFTALMOPATÍA DISTIROIDEA ASOCIADA A HIPOTIROIDISMO
}

\section{DYSTHYROID OPHTHALMOPATHY ASSOCIATED WITH HYPOTHYROIDISM}

\author{
MACIÁ-BOBES C ${ }^{1}$, RONZÓN-FERNÁNDEZ A ${ }^{2}$
}

\section{RESUMEN}

Caso clínico: La oftalmopatía por enfermedad de Graves (exoftalmos, infiltración muscular y palpebral) se asocia casi sistemáticamente a hipertiroidismo.

Paciente diagnosticada de hipotiroidismo subclínico y tratada adecuadamente con tiroxina oral. Unos meses después desarrolla un exoftalmos bilateral y simultáneamente presenta anticuerpos séricos antireceptor de TSH positivos. Se suspende entonces el tratamiento con tiroxina, y se comprueba que el hipotiroidismo ha progresado hasta hacerse primario. Se establece el diagnóstico de enfermedad de Graves hipotiroidea.

Discusión: El diagnóstico se basó en la existencia de la oftalmopatía y los anticuerpos positivos, ambos específicos de la enfermedad de Graves. La coexistencia de hipotiroidismo es excepcional, pero posible.

Palabras clave: Oftalmopatía por enfermedad de Graves, hipotiroidismo, anticuerpos anti-receptor de tirotropina, tiroxina, terapéutica.

\begin{abstract}
Clinical case: Graves' ophthalmopathy (exophthalmos, muscular and eyelid infiltration) is associated almost systematically to hyperthyroidism.

A female patient was diagnosed with subclinical hypothyroidism and treated with oral thyroxine. Months later she developed bilateral exophthalmos and was serum-positive for thyrotropin receptor antibodies. Thyroxine treatment was suspended, and it was verified that her condition had developed into primary hypothyroidism. A diagnosis of Graves' disease with hypothyroidism was made.

Discussion: The diagnosis was based on the existence of ophthalmopathy and positive serum antibodies, both specific indicators of Graves' disease. The coexistence of hypothyroidism with Graves' disease is exceptional, but possible (Arch Soc Esp Oftalmol 2007; 82: 765-768).
\end{abstract}

Key words: Graves' ophthalmopathy, hypothyroidism, thyrotropin receptor antibodies, thyroxine, therapeutics.

\footnotetext{
Recibido: 17/5/06. Aceptado: 30/10/07.

Sección de Endocrinología. Departamento de Medicina Interna. Hospital San Agustín. Avilés. Asturias. España.

1 Doctora en Medicina.

2 Licenciada en Medicina.

Correspondencia:

Carmen Maciá Bobes

Sección de Endocrinología. Hospital San Agustín

Camino de Heros, 4

33400 Avilés (Asturias)

España

E-mail: cmacb@arrakis.es
} 


\section{INTRODUCCIÓN}

La oftalmopatía por enfermedad de Graves (EG) se caracteriza por un grado variable de proptosis ocular, generalmente bilateral, y signos infiltrativos de la musculatura extrínseca del ojo o de los tejidos blandos periorbitarios. Las formas clínicamente evidentes, que cursarían con exoftalmos, quemosis, edemas palpebrales u oftalmoplejia, se presentan en un 10-20\% de los pacientes con EG, aunque las formas sutiles, demostrables en una tomografía axial computarizada por ejemplo, son mucho más prevalentes (1). De modo infrecuente esta oftalmopatía puede aparecer en ausencia de hipertiroidismo, bien asociada a normofunción tiroidea o -excepcionalmente - a hipofunción (2). Presentamos un caso de EG con hipotiroidismo y exoftalmos.

\section{CASO CLÍNICO}

Mujer de 59 años, sana, no fumadora y asintomática, remitida desde Atención Primaria a la consulta de Endocrinología en agosto de 2003 por hipotiroidismo subclínico. No presentaba bocio y dos determinaciones recientes de función tiroidea habían mostrado niveles séricos de tiroxina libre (T4L) normales y niveles de tirotropina (TSH) elevados: 13,55 y $13,86 \mathrm{mcUI} / \mathrm{mL}$ (valores normales - VN-: 0,25-4,22). Los anticuerpos anti-peroxidasa tiroidea (Ac anti-TPO) eran positivos a títulos altos. Con el diagnóstico de hipotiroidismo subclínico autoinmunitario sin bocio se inició tratamiento con $50 \mathrm{mcg} /$ día de tiroxina oral (1 $\mathrm{mcg} / \mathrm{kg}$ peso), y se comprobó en análisis realizados a los 6 y 12 meses que esta dosis de sustitución era correcta (TSH normal). En diciembre de 2004 la paciente acudió espontáneamente a la consulta para comentar que en el último mes había notado protrusión de los globos oculares, irritación conjuntival y lagrimeo. A la exploración, realizada por el Servicio de Oftalmología, se objetivó un exoftalmos bilateral moderado y una motórica ocular normal. Se estableció el diagnóstico de oftalmopatía de probable origen tiroideo (no se consideraron indicadas pruebas complementarias), se recomendó a la paciente tratamiento con lágrimas artificiales, y a la Sección de Endocrinología la investigación de una posible EG. Se determinaron entonces los niveles séricos de T4L y TSH (normales con tratamiento), Ac anti-TPO (positivos) y anticuerpos anti-receptor de TSH (Ac antiTSHr), que fueron igualmente positivos: $40 \mathrm{U} / \mathrm{L}$ (VN inferior a 10).

Puesto que estos anticuerpos son virtualmente patognomónicos de la EG, y puesto que la normofunción tiroidea en esta enfermedad es menos rara que la hipofunción, se suspendió el tratamiento con tiroxina para comprobar el estatus de la función tiroidea intrínseca (que no puede establecerse en presencia de tratamiento). Tras dos meses sin tiroxina oral la T4L era de 0,47 ng/dL (VN: 0,7-1,7) y la TSH de 54,01, por lo que la paciente fue diagnosticada de hipotiroidismo primario franco en el seno de una EG y se reintrodujo tratamiento sustitutivo. Hasta la actualidad (abril de 2006) el exoftalmos ha permanecido sin cambios (revisiones anuales en Oftalmología), y la función tiroidea — con tratamiento- es normal.

\section{DISCUSIÓN}

Al diagnóstico de EG se puede llegar por dos vías: la que parte de un paciente con hipertiroidismo, que consulta en Endocrinología, y la que parte de un paciente con oftalmopatía sugestiva, que consulta en Oftalmología. En el primer caso, la demostración de unos Ac anti-TSHr positivos en suero confirma el diagnóstico. También lo confirma la existencia de la oftalmopatía, pero ésta es poco frecuente y no suele aparecer en ausencia de anticuerpos. Cabe, por último, un diagnóstico de exclusión en sujetos hipertiroideos sin anticuerpos y sin oftalmopatía, pero sin bocio o con un bocio difuso (no nodular) (3).

En los casos en los que el síntoma-guía inicial es la oftalmopatía, se debe investigar siempre el estado de la función tiroidea. Si se comprueba hipertiroidismo la enfermedad de Graves es segura. Si no, el diagnóstico requiere la positividad de los Ac antiTSHr, en ausencia de los cuales (aunque puede tratarse de una limitación de la técnica) resulta aventurado establecer con certeza la EG. En ocasiones, con el paso del tiempo, el paciente acaba sufriendo un hipertiroidismo o se acaban positivizando anticuerpos previamente negativos (4).

Es posible la coexistencia de una oftalmopatía sugestiva de EG y unos Ac anti-TSHr positivos (es decir, de una EG), junto con un hipotiroidismo, situación que algunos autores denominan «enfer- 
medad de Graves hipotiroidea», por analogía con la EG eutiroidea. En un estudio realizado en Singapur sobre más de 1000 pacientes con este tipo de oftalmopatía se comprobó que sólo un $1,9 \%$ no eran hipertiroideos, y de ellos sólo un 0,2\% eran hipotiroideos (2). Se han publicado casos aislados de esta variante de la EG, en ocasiones con formas de oftalmopatía severa, con hipotiroidismos muy marcados y con títulos muy altos de Ac anti-TSHr (5). El mecanismo fisiopatológico implicado parece ser la capacidad de inhibición de la función tiroidea por parte de algún tipo de Ac anti-TSHr: la mayoría de estos anticuerpos son estimulantes del receptor, lo cual se traduce en hiperfunción y bocio, pero existiría un subgrupo no estimulante -que bloquearía el receptor (4). Con los métodos analíticos disponibles actualmente en la clínica habitual no es posible distinguir ambos subgrupos.

En resumen la EG con hipotiroidismo existe, aunque es excepcional, y a su diagnóstico se llega siempre a partir del estudio de función tiroidea de un paciente con oftalmopatía.

\section{BIBLIOGRAFÍA}

1. Pérez Moreiras JV, Coloma Bockos JE, Prada Sánchez MC. Orbitopatía tiroidea (fisiopatología, diagnóstico y tratamiento). Arch Soc Esp Oftalmol 2003; 78: 407-431.

2. Khoo DH, Eng PH, Ho SC, Tai ES, Morgenthaler NG, Seah LL et al. Graves'ophthalmopathy in the absence of elevated free thyroxine and triiodothyronine levels: prevalence, natural history, and thyrotropin receptor antibody levels. Thyroid 2000; 10: 1093-1100.

3. Teissier MP, Lopez S. Orbitopathie dysthyroüdienne: physiopathologie, équilibre hormonal. J Fr Ophtalmol 2004; 27: 806-809.

4. Kazuo K, Fujikado T, Ohmi G, Hosohata J, Tano Y. Value of thyroid stimulating antibody in the diagnosis of thyroid associated ophthalmopathy of euthyroid patients. $\mathrm{Br} \mathrm{J}$ Ophthalmol 1997; 81: 1080-1083.

5. Jorge Z, Nobre EL, Santana A, Castro JJ. Doença autoimune da tiroideia. Acta Med Port 2005; 18: 88-92. 